\title{
Prevalence and molecular characterization of novel recombinant enterovirus $G$ isolates in Jeju Province of South Korea
}

\author{
Ji Hyun Jeon, Changhee Lee* \\ Animal Virology Laboratory, School of Life Sciences, BK21 Plus KNU Creative BioResearch Group, \\ Kyungpook National University, Daegu 41566, Korea
}

\begin{abstract}
Enterovirus species G(EV-G) is highly diverse, and is ubiquitous in pig populations, usually without diarrhea. The present study aimed to investigate the presence of novel EV-G recombinants with the torovirus papain-like cysteine protease (PLCP) in Jeju pig herds. EV-G1-PLCP mono-infections were most prevalent in diarrheic weaned piglets. The PLCP genes of the Jeju isolates varied in size and junction sequences, and were greatly heterogeneous, with $77.0-90.7 \%$ homology amongst all recombinants. Our results suggest that the exogenous PLCP gene has undergone continuous rapid mutation in the individual EV-G genomes following crossorder recombination, thereby causing clinical disease in swine.
\end{abstract}

Keywords: cross-order recombination, EV-G, PLCP, porcine diarrhea, torovirus

*Corresponding author

Changhee Lee

Animal Virology Laboratory, School of

Life Sciences, BK21 Plus KNU Creative

BioResearch Group, Kyungpook National

University, 80 Daehak-ro, Buk-gu, Daegu

41566, Korea

Tel: $+82-53-950-7365$

Fax: $+82-53-955-5522$

E-mail: changhee@knu.ac.kr

ORCID:

Ji Hyun Jeon

https://orcid.org/0000-0001-7345-6613

Changhee Lee

https://orcid.org/0000-0002-5930-5461

Conflict of Interest

The authors declare no conflicts of interest.

Received: March 20, 2019

Revised: May 10, 2019

Accepted: May 28, 2019
Enteroviruses (EVs) are small, naked, single-strand, positive-sense RNA viruses which are associated with numerous human and mammalian diseases. They belong to the genus Enterovirus in the family Picornaviridae of the order Picornavirales. The EV genus consists of 15 species including EV A-L and rhinovirus $\mathrm{A}-\mathrm{C}$, and infect humans (species $\mathrm{A}-\mathrm{D}$ ), cows (species $\mathrm{E}$ and F), swine (species $G$ ), and nonhuman primates (A, B, D, H, and J) [1]. The EV genome is approximately $7.5-\mathrm{kb}$ in length and contains a single open reading frame, flanked by $5^{\prime}$ - and $3^{\prime}$-untranslated regions and a $3^{\prime}$ poly (A) tail, which encodes a large polyprotein that is proteolytically matured to produce 4 structural (viral protein [VP] $1-4)$ and 7 nonstructural $\left(2 \mathrm{~A}^{\text {pro }}, 2 \mathrm{~B}\right.$, $2 \mathrm{C}, 3 \mathrm{~A}, 3 \mathrm{~B}, 3 \mathrm{C}^{\mathrm{pro}}$, and $\left.3 \mathrm{D}^{\mathrm{pol}}\right)$ viral proteins [2].

EV species $\mathrm{G}(\mathrm{EV}-\mathrm{G})$ includes a heterogeneous group of 20 genotypes that undergo continuous intratypic and intertypic recombination events [1,3,4]. EV-G infections have been frequently detected among the swine populations in multiple pig-raising countries [3-5]. In spite of their genetic diversity and high prevalence, EV-Gs are generally considered to be irrelevant to clinical disease in pigs. However, recent studies have reported novel EV-G1, -G2, and -G17 variants in fecal samples from porcine diarrheic diseases in the United States, Belgium, and Japan. These recombinant viruses, namely type 1, are identical in the insertion of a porcine torovirus (ToV)-derived papain-like cysteine protease $(\mathrm{PLCP})$ gene at the $2 \mathrm{C} / 3 \mathrm{~A}$ junction region, as a result of a natural cross-order recombination between EV-G (order Picornavirales) and ToV (order Nidovirales) [3,6-8]. More recently, the second class (type 2) of a recombinant capsid-null EV-G strain, which carries the PLCP gene replacing the entire viral capsid protein region, has been identified in Chinese pig farms [9]. Since the ToV-PLCP gene inserted into the recombinant EV-G genome is known to act as an innate immune antagonist, the horizontal acquisition of the nidoviral gene plays an important role in viral pathogenesis [8].

In early 2018, the recombinant EV-G1-PLCP strain was discovered in mainland South Korea to be a sole enteric viral pathogen in clinically diarrheic weaned piglets [10]. Genetic and phylogenetic analyses revealed that the Korean EV-G1-PLCP strain is classified into the G1 genotype, but its inserted PLCP is most closely related to that of the recombinant G17 genotype. More interestingly, the size and junction sequence of the exogenous PLCP gene var- 
A

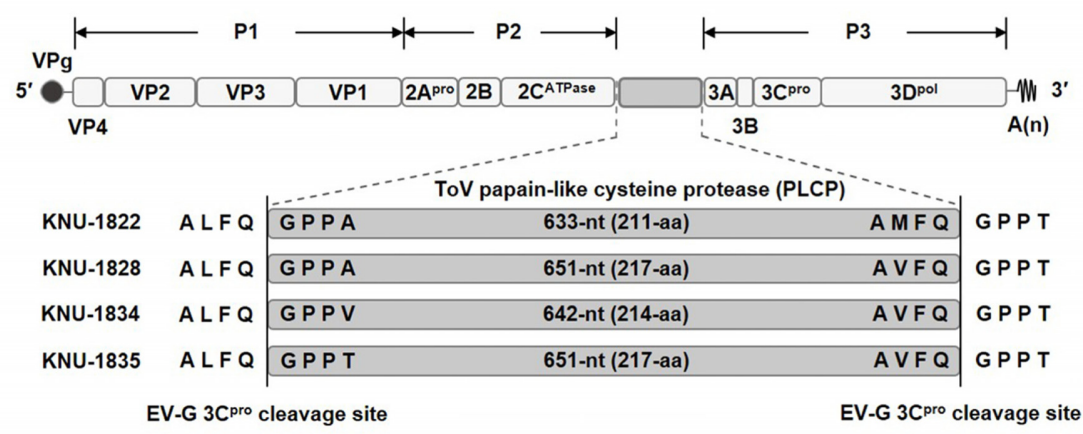

B
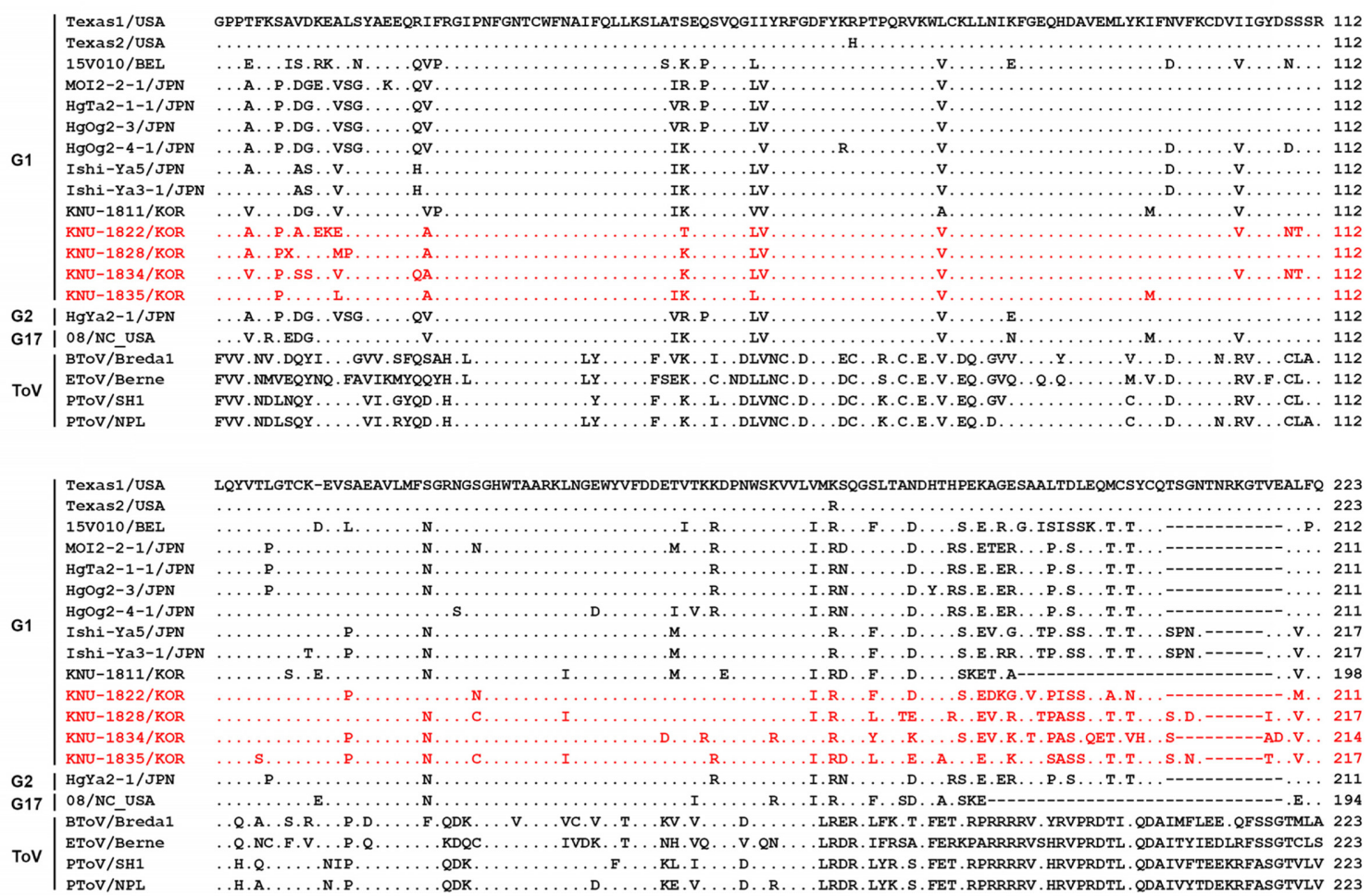

Fig. 1. Schematic representation of the genome organization of EV-G1-PLCP. (A) The Jeju EV-G1-PLCP genome contains a single open reading frame flanked by a long 5' UTR of 813-nt and a short 3' UTR of 71-nt, followed by a poly (A) tail (An). Vertical lines on top indicate the polyprotein processing sites which create the precursor polyproteins $\mathrm{P} 1, \mathrm{P} 2$, and $\mathrm{P} 3$ using EV-G $3 \mathrm{C}^{\text {pro }}$. The porcine ToV PLCP gene is presented as a shaded box at the $2 \mathrm{C} / 3 \mathrm{~A}$ cleavage junction. The $5^{\prime}$ - and $3^{\prime}$-boundary sequences of the $3 \mathrm{C}^{\text {pro }}$ cleavage sites are shown as enlarged, shaded boxes. (B) Multiple alignment of the aa sequences of the PLCP regions of the recombinant EV$\mathrm{G}$ and ToV strains. The Jeju recombinant EV-G1-PLCP strains identified in this study are marked with a red color. Dots denote amino acids (aa) identical to the Texas1/USA sequence, and dashes indicate deleted sequences.

EV-G1, enterovirus species G1; UTR, untranslated region; ToV, torovirus; PLCP, papain-like cysteine protease; 3C ${ }^{\text {pro }}, 3 \mathrm{C}$ protease; nt, nucleotide.

ies among recombinant strains, suggesting spatially independent evolution in different geographical areas [10]. In this study, we sought to further explore the prevalence of EV-GPLCP in clinical cases associated with diarrhea in swine farms in Jeju Province, the largest island situated $130 \mathrm{~km}$ off the southern coast of mainland South Korea, and to perform full-length genome sequence analysis.

Small intestine or stool samples $(n=107)$ were independently collected from pigs with diarrhea at 33 different farms located on Jeju Island in the period from January through to December 2018, and were tested for the detection of pig scours-associated viruses including EV-G, swine enteric coronaviruses, and rotavirus, using reverse transcription polymerase chain reaction with virus-specific primers [10]. Of the 107 porcine fecal and intestinal samples used in our EV-G-PLCP survey, 11 samples (10.3\%) from 4 different 


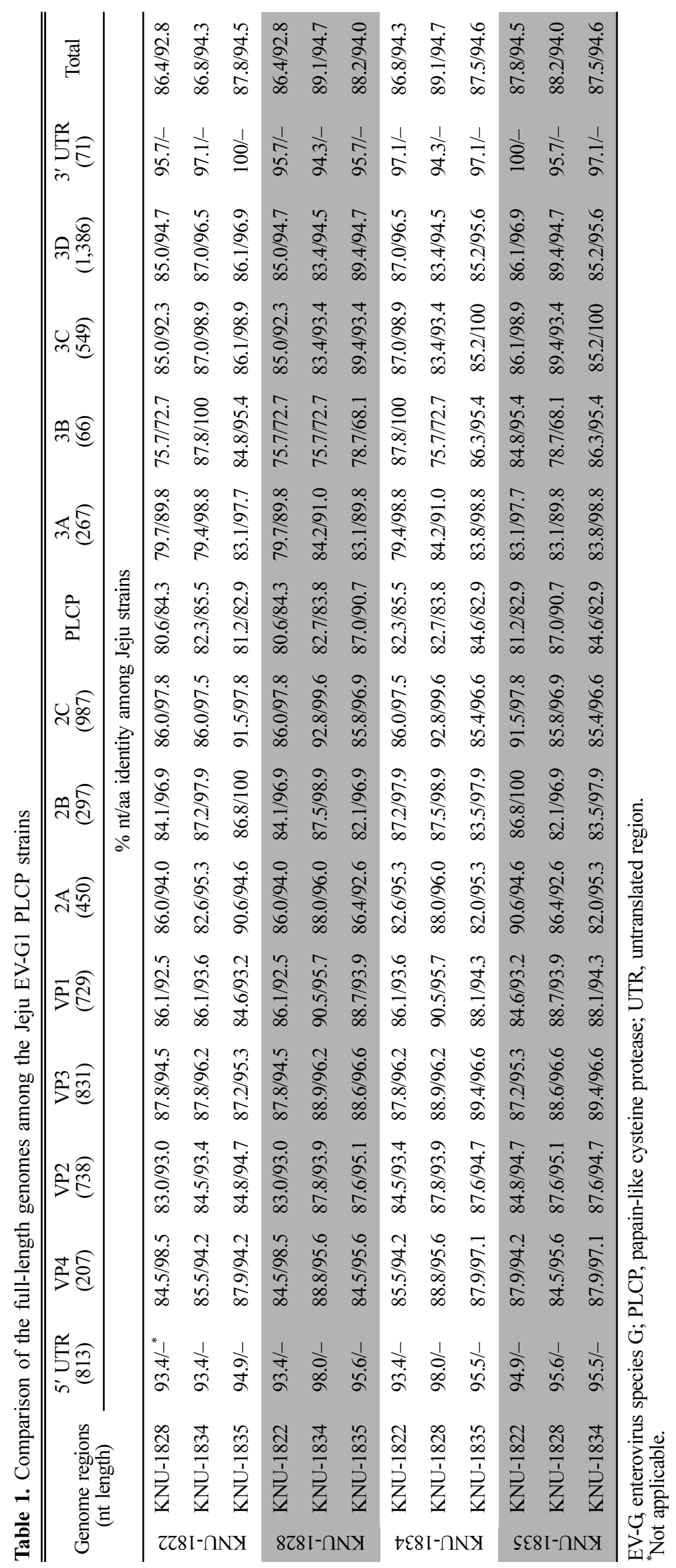


farms tested positive. Ten samples were confirmed as EV-GPLCP mono-infections, while the remaining EV-G-PLCPdetected sample was found to be positive for porcine epidemic diarrhea virus (PEDV). All EV-G-PLCP recombinants were identified from 21-60 day-old piglets on commercial farrowto-finish farms that have suffered from small-scale outbreaks of post-weaning diarrhea. The four Jeju isolates representing individual pig farms were designated as KNU-1822, -1828, -1834 , and -1835 . Sequences of approximately 1,200-nucleotide (nt) encompassing the inserted region flanked by the EV-G
$2 \mathrm{C}$ and $3 \mathrm{~A}$ genes from all of the isolates were determined using by the traditional Sanger sequencing method.

Initial nucleotide sequencing identified the presence of exogenous sequences with different lengths at the $2 \mathrm{C} / 3 \mathrm{~A}$ junction of the enterovirus genome, and revealed genetic diversity among the KNU strains with $9.3-17.1 \%$ sequence variation (Fig. 1A, Supplementary Table 1). A BLAST search against the GenBank nt sequence database found that these distinctive foreign sequences are closely homologous to the PLCP sequence variants in type 1 recombinant EV-G strains.
A

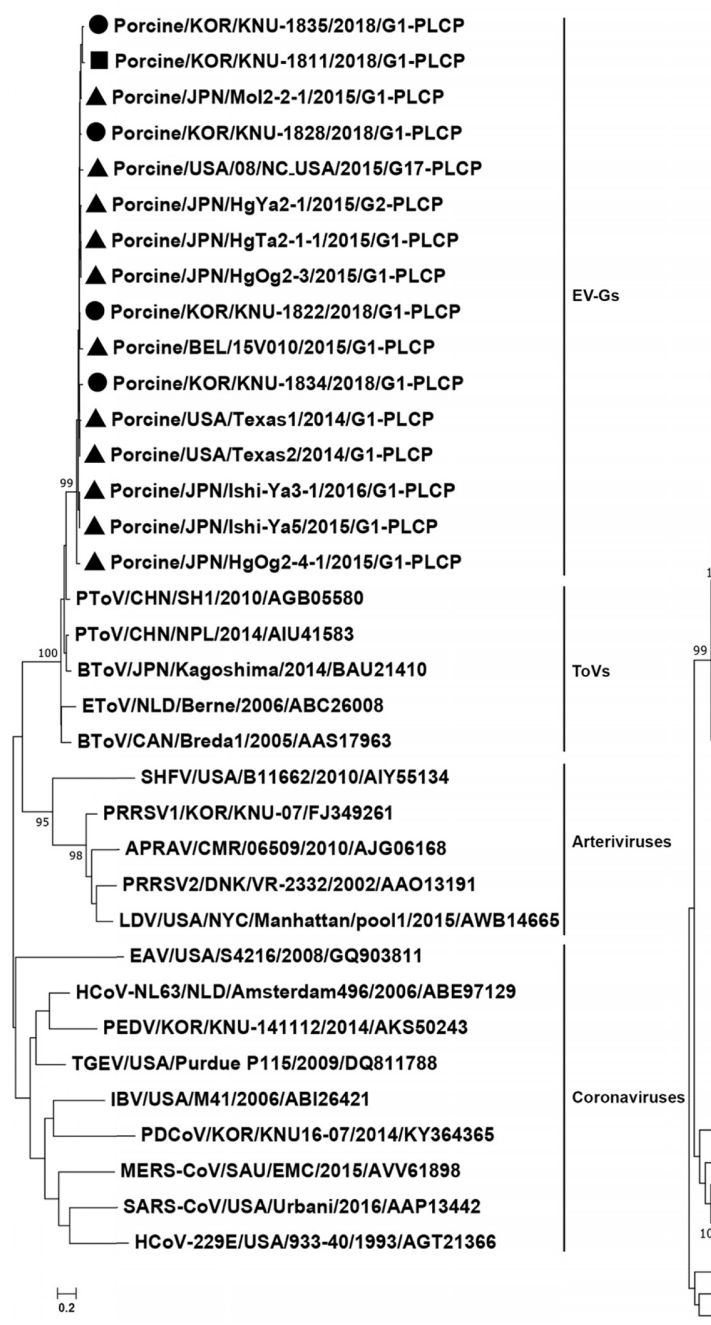

B

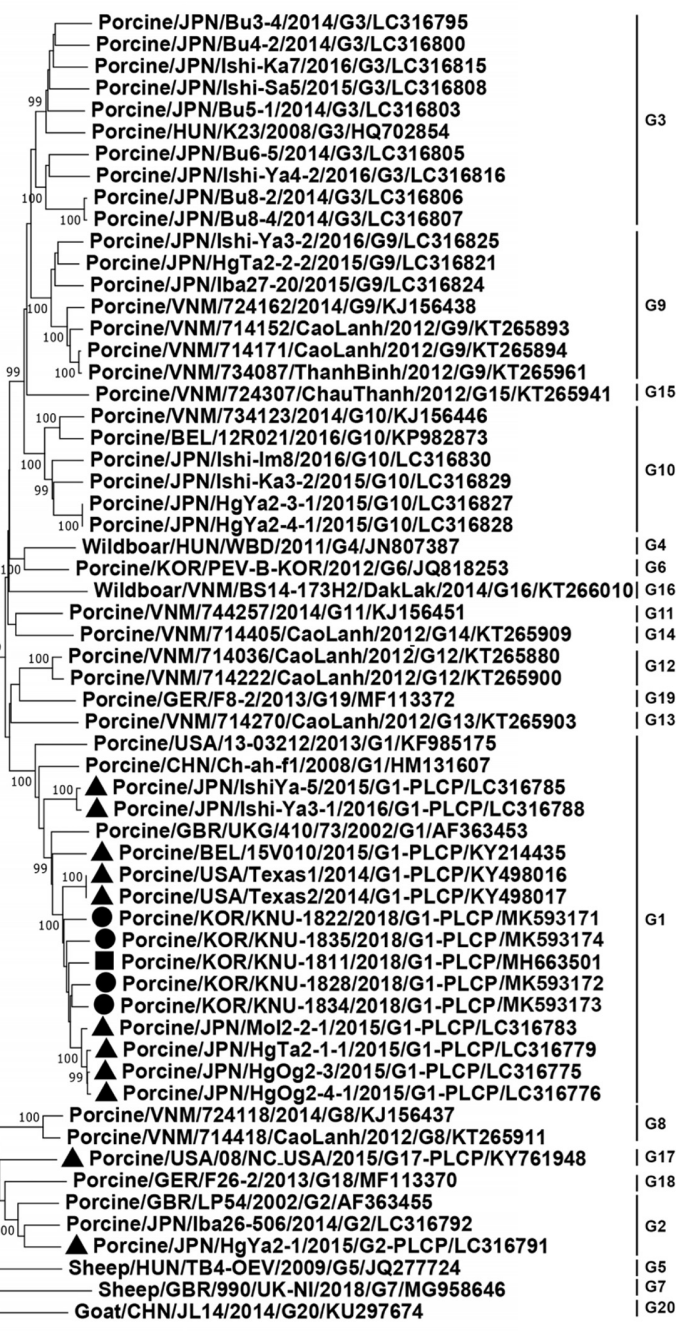

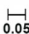

Fig. 2. Phylogenetic analysis based on the PLCP genes of EV-Gs and nidoviruses, including ToVs, arteriviruses, and coronaviruses (A) and the VP1 genes of EV-G genotypes (B). Multiple sequence alignments were created using ClustalX 2.0, and the phylogenetic trees were constructed from the aligned nucleotide (nt) sequences using the neighbor-joining method. Numbers at each branch represent bootstrap values greater than $50 \%$ of 1,000 replicates. Hosts of origin, geographical origins, names of the strains, years of isolation, genotypes, and GenBank accession numbers are shown. The EV-G genotypes are indicated on the right-hand side in panel B. Solid circles denote the Jeju recombinant EV-G1-PLCP strains identified in this study; a solid square indicates the mainland Korean EV-G1-PLCP strain identified in a previous study; solid triangles denote global EV-G PLCP strains reported in previous studies. Scale bars indicate nt substitutions per site.

EV-G, enterovirus species G; PLCP, papain-like cysteine protease; ToV, torovirus. 
The length of the inserted PLCP sequences differed among the recombinant Jeju strains, with lengths of 633-nt in KNU1822, 651-nt in KNU-1828, 642-nt in KNU-1834, and 651-nt in KNU-1835. These are also different from previously identified strains from a range of geographical areas: 582/669-nt in the US; 636-nt in Belgium; 633/651-nt in Japan; and 594nt in mainland Korea (Fig. 1B). The PLCP sequences of the Jeju EV-G strains only shared $77.0-88.0 \%$ amino acid (aa) sequence identity with global recombinant EV-G1, -G2, and G17 strains, with the highest similarity being to the Japanese EV-G1 strain JPN/Ishi-Ya-5/2015 [3]. The exogenous PLCP gene serves as a functional viral component produced in cells infected with the recombinant EV-G virus by trimming the $2 \mathrm{C} / 3 \mathrm{~A}$ junction of the polyprotein at cleavage sequences, such as ALFQ $\downarrow$ GPPT, recognized by the viral $3 C^{\text {pro }}$ protease [8]. On the basis of sequence similarity, numerous putative cleavage sequences have been identified in global strains, which involve GPPT-ALFQ, GPPA-ALFQ, GPPE-ALPQ, GPPV-AEFQ, and GPPV-AVFQ [3,6-8,10]. The PLCP genes of the chimeric Jeju strains consistently contained their own predicted $3 \mathrm{C}^{\text {pro }}$ cleavage sites, including GPPA-AMFQ, GPPA-AVFQ, GPPV-AVFQ, and GPPT-AVFQ at the Nand C-termini (Fig. 1A). Subsequent phylogenetic analysis based on the PLCP genes of EV-Gs and nidoviruses showed that the exogenous PLCP genes of the Jeju strains are most closely related to those of other global recombinant EV-G strains, forming a well-defined cluster, but are distantly related to those of porcine, bovine, and equine ToVs with lower aa sequence identities (42.6-55.1\%) (Fig. 2A).
To investigate their genetic relatedness to previously reported domestic and global strains, the complete genomes of all Jeju chimeric EV-G isolates were sequenced using Sanger sequencing as described previously [10]. The fulllength genomic sequences were assembled and deposited in the GenBank database under the accession numbers listed in Fig. 2B. The genomic and VP1 sequences of the Jeju strains shared $72.1-72.9 \%$ nt and $84.0-86.1 \%$ aa identity with those of the prototype EV-G strain PEV9/UKG/410/73. Compared with other chimeric EV-G1, -G2, and -G17 strains, the Jeju isolates showed relatively high sequence homology (83.8$87.5 \%$ nt and $88.2-96.8 \%$ aa identity at the genome and VP1 gene levels) with the US, Belgium, Japanese, and mainland Korean EV-G1 strains, but had lower identities (79.5-80.5\% nt and 55.3-63.2\% aa) at the genome and VP1 levels with the Japanese EV-G2 and US EV-G17 strains. Details of the sequence similarities, at both nt and aa levels, between the $\mathrm{KNU}$ isolates and prototypic or recombinant EV-G strains are summarized in Supplementary Tables 2 and 3.

Phylogenetic analysis based on the EV-G VP1 sequences revealed that the Jeju KNU strains grouped with other global recombinant EV-G1-PLCP strains within the G1 genotype (Fig. 2B). Whole-genome phylogeny also showed a distinct clade composed exclusively of G1-PLCP recombinants (Fig. 3A). All of the G1-PLCP strains, including the Jeju isolates, were phylogenetically distant from the original wild-type G1 strains, but were adjacent to the G3 and G10 genotypes, implying that they may be derived from a common ancestor. A phylogenetic tree constructed from whole-genome data,

Table 3. Comparison of the full-length genomes of the Jeju isolates and representative EV-G strains

\begin{tabular}{|c|c|c|c|c|c|c|c|c|c|c|c|}
\hline \multirow{2}{*}{$\begin{array}{l}\text { Strain name } \\
\text { (genotype) }\end{array}$} & \multicolumn{11}{|c|}{ Nucleotide identity (\%) (No. of nucleotide difference) } \\
\hline & UKG & Texas1 & $15 \mathrm{~V} 010$ & MOI2-2-1 & HgYa2-1 & 08_NC & KNU-181 & KNU-1822 & KNU-1828 & KNU-183 & KNU-1835 \\
\hline UKG (G1) & & $\begin{array}{c}71.4 \\
(2,303)\end{array}$ & $\begin{array}{c}73.1 \\
(2,160)\end{array}$ & $\begin{array}{c}73.1 \\
(2,154)\end{array}$ & $\begin{array}{c}68.5 \\
(2,524)\end{array}$ & $\begin{array}{c}69.4 \\
(2,439)\end{array}$ & $\begin{array}{c}73.1 \\
(2,143)\end{array}$ & $\begin{array}{c}73.2 \\
(2,151)\end{array}$ & $\begin{array}{c}72.3 \\
(2,229)\end{array}$ & $\begin{array}{c}72.7 \\
(2,192)\end{array}$ & $\begin{array}{c}72.7 \\
(2,196)\end{array}$ \\
\hline Texas1 (G1) & & & $\begin{array}{c}82.6 \\
(1,399)\end{array}$ & $\begin{array}{c}84.9 \\
(1,208)\end{array}$ & $\begin{array}{c}78.9 \\
(1,698)\end{array}$ & $\begin{array}{c}77.9 \\
(1,777)\end{array}$ & $\begin{array}{c}85.0 \\
(1,200)\end{array}$ & $\begin{array}{c}84.6 \\
(1,237)\end{array}$ & $\begin{array}{c}84.3 \\
(1,263)\end{array}$ & $\begin{array}{c}84.3 \\
(1,260)\end{array}$ & $\begin{array}{c}84.6 \\
(1,236)\end{array}$ \\
\hline 15V010 (G1) & & & & $\begin{array}{c}83.9 \\
(1,290)\end{array}$ & $\begin{array}{c}80.2 \\
(1,587)\end{array}$ & $\begin{array}{c}79.5 \\
(1,647)\end{array}$ & $\begin{array}{c}83.3 \\
(1,339)\end{array}$ & $\begin{array}{c}84.1 \\
(1,273)\end{array}$ & $\begin{array}{c}84.2 \\
(1,270)\end{array}$ & $\begin{array}{c}84.4 \\
(1,252)\end{array}$ & $\begin{array}{c}83.8 \\
(1,303)\end{array}$ \\
\hline MOI2-2-1 (G1) & & & & & $\begin{array}{c}82.9 \\
(1,366)\end{array}$ & $\begin{array}{c}79.5 \\
(1,640)\end{array}$ & $\begin{array}{c}86.5 \\
(1,076)\end{array}$ & $\begin{array}{c}86.3 \\
(1,092)\end{array}$ & $\begin{array}{c}86.7 \\
(1,062)\end{array}$ & $\begin{array}{c}87.2 \\
(1,025)\end{array}$ & $\begin{array}{c}85.8 \\
(1,134)\end{array}$ \\
\hline HgYa2-1 (G2) & & & & & & $\begin{array}{c}81.3 \\
(1,499)\end{array}$ & $\begin{array}{c}79.7 \\
(1,627)\end{array}$ & $\begin{array}{c}80.2 \\
(1,586)\end{array}$ & $\begin{array}{c}80.5 \\
(1,565)\end{array}$ & $\begin{array}{c}80.4 \\
(1,574)\end{array}$ & $\begin{array}{c}79.5 \\
(1,649)\end{array}$ \\
\hline 08_NC (G17) & & & & & & & $\begin{array}{c}80.6 \\
(1,546)\end{array}$ & $\begin{array}{c}80.3 \\
(1,577)\end{array}$ & $\begin{array}{c}80.1 \\
(1,598)\end{array}$ & $\begin{array}{c}80.4 \\
(1,569)\end{array}$ & $\begin{array}{c}79.5 \\
(1,651)\end{array}$ \\
\hline KNU-1811 (G1) & & & & & & & & $\begin{array}{c}85.9 \\
(1,127)\end{array}$ & $\begin{array}{c}86.6 \\
(1,070)\end{array}$ & $\begin{array}{c}87.5 \\
(1,004)\end{array}$ & $\begin{array}{c}85.9 \\
(1,131)\end{array}$ \\
\hline KNU-1822 (G1) & & & & & & & & & $\begin{array}{c}86.4 \\
(1,093)\end{array}$ & $\begin{array}{c}86.8 \\
(1,054)\end{array}$ & $\begin{array}{l}87.8 \\
(974)\end{array}$ \\
\hline KNU-1828 (G1) & & & & & & & & & & $\begin{array}{c}89.1 \\
(869)\end{array}$ & $\begin{array}{c}88.2 \\
(943)\end{array}$ \\
\hline KNU-1834 (G1) & & & & & & & & & & & $\begin{array}{c}87.5 \\
(998)\end{array}$ \\
\hline
\end{tabular}

EV-G, enterovirus species G. 
excluding the PLCP sequence, in all chimeric strains also showed the comparable grouping structure (Fig. 3B). These data suggested that genetic alterations to EV-G, including random mutations throughout the entire genome and a gene insertion at a specific site, have simultaneously arisen, leading to independent evolution to the same genotype carrying the foreign gene or other genotypes.

In summary, the prevalence of a type 1 recombinant EV-GPLCP virus characterized by the recombination of an exogenous porcine ToV-like PLCP gene into the $2 \mathrm{C} / 3 \mathrm{~A}$ junction region of the EV-G genome in diarrheal samples from pigs was confirmed on Jeju Island, South Korea. To the best of our knowledge, this is the first study to the molecular epidemiology of EV-G recombinants in Jeju pig herds. Our study revealed that all incidents occurred in weaned piglets and,

A

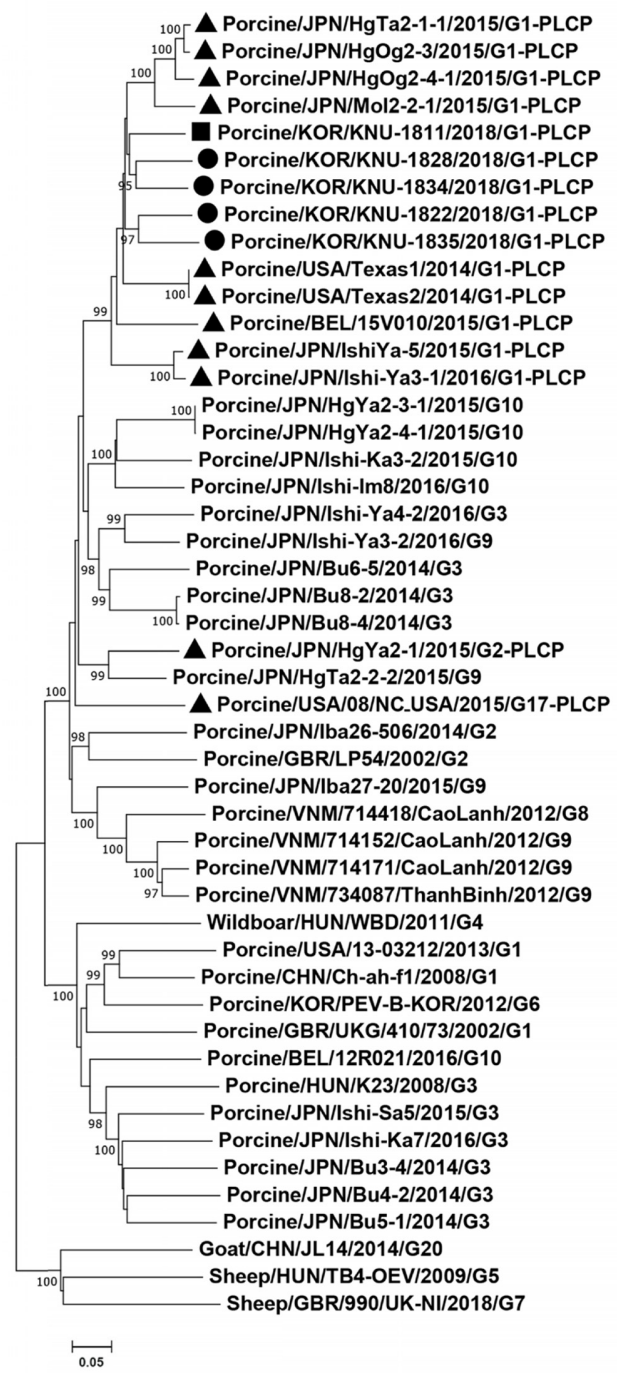

except for one co-infection with PEDV, all cases were type 1 EV-G1-PLCP mono-infections, indicating that there is an association of this recombinant with post-weaning diarrhea. Further study is need to expand our molecular-based survey, in order to investigate the incidence of EV-G PLCP variants and to identify the presence of type 2 recombinant EV-Gs in Korean pig populations. VP1-based sequencing and phylogenetic analyses revealed that the Jeju recombinant G1 strains are closely related to each other, having $92.5-95.7 \%$ homology, and are also similar to previously reported recombinant G1 strains, with $88.2-96.8 \%$ homology. However, the inserted PLCP genes of the Jeju strains shared relatively low, variable similarities with each other (82.9-90.7\% homology) and other recombinant strains $(77.0-88.0 \%$ homology), indicating the heterogeneity of the foreign PLCP. These strains are

B

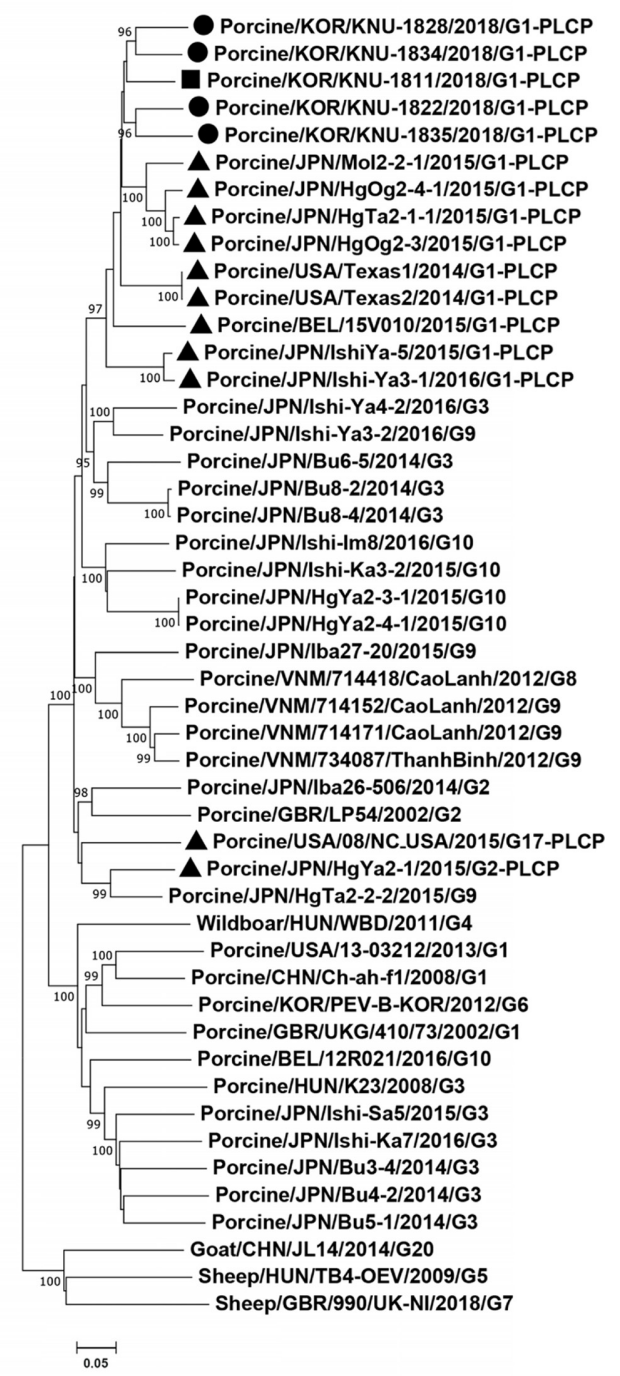

Fig. 3. Phylogenetic analysis based on the full-length genomic sequences: (A) including; (B) excluding the insertion PLCP sequences of EV-G strains. Solid circles denote the Jeju recombinant EV-G1-PLCP strains; a solid square indicates the mainland Korean EVG1-PLCP strain; solid triangles denote global EV-G PLCP strains. Scale bars indicate nucleotide substitutions per site. EV-G, enterovirus species G; PLCP, papain-like cysteine protease. 
distantly related to the mainland G1-PLCP strain KNU-1811, which has previously been shown to have the highest PLCP identity to the US G17-PLCP strain [10]. The sizes of the recombinant EV-G PLCP sequences and their junction sequences are highly heterogeneous between individual strains, suggesting independent viral evolution. Collectively, these findings indicate that genetic drift has more independently and quickly proceeded in the exogenous PLCP sequence compared with the endogenous viral sequence after its well-organized insertion into the dominant EV-G genome. Although horizontal viral gene transfer (e.g., cross-order recombination) occurs infrequently, this event-derived EV-G-PLCP variants seem to emerge frequently in the field. Furthermore, a growing body of evidence suggests that the lateral acquisition of heterologous immune antagonist genes such as PLCP might serve as a novel strategy for host immune evasion of EV-Gs, increasing their pathogenic potential under various field situations. Future work should be directed towards obtaining a recombinant EV-G isolate that can grow efficiently in cell culture, in order to evaluate it as a possible causative agent of porcine diarrheal diseases. Our study advances the understanding of the genetic evolution of EV-G driven by an extraordinary but precise viral heterologous recombination, and supports the notion that this uncommon event allows one of the most prevalent but asymptomatic viruses in porcine feces to crossly gain an exotic virus-encoded anti-immune component from an entirely heterogeneous viral family, which continually experiences rapid genetic drift for viral fitness and long-term survival in its natural host, ultimately transforming non-diarrhea causing EV-G into clinical disease-causing strains.

\section{Acknowledgment}

We would like to acknowledge Veterinary Research Institute at Jeju Special Self-Governing Province for providing clinical samples. This research was supported by a fund (Project Code No. Z-1543056-2016-17-01) by Research of Animal and Plant Quarantine Agency, South Korea.

\section{References}

1. Knowles NJ, Hovi T, Hyypi T, King AMQ, Lindberg AM, Pallansch MA, Palmenberg AC, Simmonds P, Skern T,
Stanway G, Yamashita T, Zell R. Picornaviridae. In: King AMQ, Adams MJ, Carstens EB, Lefkowitz EJ (eds.). Virus Taxonomy: Classification and Nomenclature of Viruses: Ninth Report of the International Committee on Taxonomy of Viruses. pp. 1221-1234, Elsevier Academic Press, London, 2012.

2. Semler BL, Wimmer E. Molecular Biology of Picornaviruses. pp. 502, ASM press, Washington, D.C., 2002.

3. Tsuchiaka $S$, Naoi $Y$, Imai R, Masuda T, Ito M, Akagami M, Ouchi Y, Ishii K, Sakaguchi S, Omatsu T, Katayama Y, Oba M, Shirai J, Satani Y, Takashima Y, Taniguchi Y, Takasu M, Madarame H, Sunaga F, Aoki H, Makino S, Mizutani T, Nagai M. Genetic diversity and recombination of enterovirus $\mathrm{G}$ strains in Japanese pigs: high prevalence of strains carrying a papain-like cysteine protease sequence in the enterovirus G population. PLoS One 2018; 13:e0190819.

4. Van Dung N, Anh PH, Van Cuong N, Hoa NT, CarriqueMas J, Hien VB, Campbell J, Baker S, Farrar J, Woolhouse ME, Bryant JE, Simmonds P. Prevalence, genetic diversity and recombination of species $G$ enteroviruses infecting pigs in Vietnam. J Gen Virol 2014;95:549-556.

5. Vilar MJ, Peralta B, García-Bocanegra I, Simon-Grif M, Bensaid A, Casal J, Segalés J, Pina-Pedrero S. Distribution and genetic characterization of Enterovirus $G$ and Sapelovirus A in six Spanish swine herds. Virus Res 2016;215:42-49.

6. Conceição-Neto N, Theuns S, Cui T, Zeller M, Yinda CK, Christiaens I, Heylen E, Van Ranst M, Carpentier S, Nauwynck HJ, Matthijnssens J. Identification of an enterovirus recombinant with a torovirus-like gene insertion during a diarrhea outbreak in fattening pigs. Virus Evol 2017;3:vex024.

7. Knutson TP, Velayudhan BT, Marthaler DG. A porcine enterovirus $G$ associated with enteric disease contains a novel papain-like cysteine protease. J Gen Virol 2017;98:1305-1310.

8. Shang P, Misra S, Hause B, Fang Y. A naturally occurring recombinant enterovirus expresses a torovirus deubiquitinase. J Virol 2017;91:e00450-e17.

9. Wang Y, Zhang W, Liu Z, Fu X, Yuan J, Zhao J, Lin Y, Shen Q, Wang X, Deng X, Delwart E, Shan T, Yang S. Full-length and defective enterovirus $G$ genomes with distinct torovirus protease insertions are highly prevalent on a Chinese pig farm. Arch Virol 2018;163:2471-2476.

10. Lee S, Lee C. First detection of novel enterovirus G recombining a torovirus papain-like protease gene associated with diarrhoea in swine in South Korea. Transbound Emerg Dis 2019;66:1023-1028. 